\title{
Acepromazine Maleate
}

National Cancer Institute

\section{Source}

National Cancer Institute. Acepromazine Maleate. NCI Thesaurus. Code C76652.

The maleate salt of acepromazine, a phenothiazine derivative with depressant effect on the central nervous system. Acepromazine acts as a dopamine receptor antagonist in the CNS, thereby causing sedation, muscular relaxation and a reduction in spontaneous activity. Its fast-acting neuroleptic effect with low toxicity is of particular value in veterinary medicine. 